\title{
Circulação inter e intracoletiva em Grupos de Pesquisa de História da Educação Matemática
}

\section{Inter - and intracollective circulation in the research groups of History of Mathematics Education}

\author{
Yohana Taise Hoffmann ${ }^{1}$ \\ https:// orcid.org/0000-0002-3590-315X \\ David Antonio da Costa ${ }^{2}$ \\ https://orcid.org/0000-0003-4493-9207 \\ Luiz R. Nakamura ${ }^{3}$ \\ https://orcid.org/0000-0002-7312-2717 \\ Demétrio Delizoicov Neto ${ }^{2}$ \\ https://orcid.org/0000-0002-5970-3400
}

\begin{abstract}
Resumo: A partir dos estudos iniciais a respeito da epistemologia de Ludwik Fleck, os conceitos como coletivos e estilos de pensamento, círculos exotéricos e esotéricos, e circulação inter e intracoletiva são relacionados aos pesquisadores de Santa Catarina que investigam a História da educação matemática (HEM): Ensino de Ciências Naturais e Matemática da Fundação Universidade Regional de Blumenau (FURB); Grupo de Estudos e Pesquisa em Educação Matemática (GEPEM) do Instituto Federal de Santa Catarina (IFSC); Grupo de Estudos Contemporâneos e Educação Matemática (GECEM) da Universidade Federal de Santa Catarina (UFSC); e o Grupo de Pesquisa de História da Educação Matemática de Santa Catarina (GHEMAT-SC) da UFSC. Neste artigo abordam-se as interações entre esses grupos, por meio de distintos modos, os quais formam uma rede em que circulam diferentes estilos de pensamento intracoletivos. Os eventos na área da HEM proporcionam a expansão e o fortalecimento dos Grupos que a estudam.

Palavras-chave: Epistemologia. Grupo de pesquisa. História da educação matemática.

Abstract: Following the initial studies on the epistemology by Ludwik Fleck (1896-1961), concepts such as collectives and thought style, exoteric and esoteric circles, and inter- and intracollectiveness, are related to the research groups in Santa Catarina that investigate the History of Mathematics Education (HEM): Teaching of Natural Sciences and Mathematics at Fundação Universidade Regional de Blumenau (FURB); Group of Studies and Research in Mathematics Education (GEPEM IFSC) of Instituto Federal de Santa Catarina (IFSC); Group of Contemporary Studies and Mathematics Education (GECEM) of Universidade Federal de Santa Catarina (UFSC); and the Research Group on the History of Mathematics Education - Santa Catarina (GHEMAT / SC) of UFSC. In this paper, we discuss the interactions, through different approaches, among these groups, which form a network of researchers, in which different intracollective thought styles circulate. Events in HEM provide the expansion and strengthening of groups that study this subject.
\end{abstract}

Keywords: Epistemology. Inter and intracollective circulation. Research group. History of Mathematics Education.

\footnotetext{
${ }^{1}$ Universidade Federal de Santa Catarina (UFSC), Programa de Pós-graduação em Educação Científica e

Tecnológica, Florianópolis, SC, Brasil. E-mail: yohanath@posgrad.ufsc.br

${ }^{2}$ Universidade Federal de Santa Catarina (UFSC), Departamento de Metodologia de Ensino, Florianopolis, SC, Brasil.

${ }^{3}$ Universidade Federal de Santa Catarina (UFSC), Departamento de Informática e Estatística, Florianopolis, SC, Brasil.
} 


\section{Apresentação}

O presente artigo emergiu a partir dos estudos iniciais a respeito da epistemologia de Ludwik Fleck (1896-1961) no Programa de Pós-Graduação em Educação Científica e Tecnológica (PPGECT) na Universidade Federal de Santa Catarina (UFSC), que instigou relacionar os conceitos deste autor como, por exemplo, coletivo de pensamento (CP), estilo de pensamento (EP), círculos exotéricos e esotéricos, e circulação inter e intracoletiva com os Grupos de pesquisa de Santa Catarina que investigam a História da educação matemática (HEM).

Nos últimos anos no campo da HEM houve um aumento do número de trabalhos, eventos (nacionais e internacionais), revistas e grupos de pesquisas. Segundo Brito e Miorim, os trabalhos de investigação, como teses e dissertações, acerca da HEM começaram com a criação dos Programas de Pós-Graduação em Educação no Brasil, a partir da interlocução de professores de Matemática com a História da Educação.

A primeira dissertação que teve a HEM como objeto de investigação se intitula "Estudo da evolução do ensino secundário no Brasil e no estado do Paraná com ênfase na disciplina de matemática" de Maria Antonieta Meneghini Martins, do ano de 1984, no Programa de Pós-Graduação em Educação pela Universidade Federal do Paraná (UFPR). A primeira tese foi em 1995, denominada "O ensino de matemática: evolução e modernização", de Maria Ângela Miorim, da Faculdade de Educação da Universidade Estadual de Campinas (Unicamp). No entanto, a produção de teses e dissertações sobre a HEM se consolidou a partir de 2007. Outro aspecto que contribuiu para a ampliação da produção nessa área são os surgimentos de vários grupos de pesquisa nacionais. (BRITO; MIORIM, 2016).

De acordo com Valente (2017, p. 613-614), a construção da comunidade de cientistas que trabalham em torno da problemática da HEM pode ser observada, também, nos eventos e revistas da área

[...] desde pelo menos o ano de 2011 é a criação de eventos nacionais e internacionais voltados à discussão de estudos sobre história da educação matemática. Cite-se como exemplos: a criação do ENAPHEM Encontro Nacional de História da Educação Matemática, em 2011, estando já na sua terceira edição; o surgimento do CIHEM - Congresso Ibero-americano de História da Educação Matemática que, em 2017, estará na sua quarta realização, na cidade de Murcia, Espanha. Também: as ICHME - International Conference on the History of Mathematics Education, que realizou, entre 19 e 22 de setembro, a sua quinta edição, em Utrecht, na Holanda.

Para além dos encontros científicos de história da educação matemática, ajunte-se à rede comunicacional a criação de, pelo menos, duas revistas especializadas: a HISTEMAT - Revista de História da Educação Matemática, órgão da Sociedade Brasileira de História da Matemática, e o International Journal for the History of Mathematics Education que circulou entre 2006 e 2016. 
O primeiro Encontro Nacional de História da Educação Matemática (ENAPHEM) ocorreu entre os dias 1 e 3 de novembro de 2012 na Universidade Estadual do Sudoeste da Bahia (UESB), no município de Vitória da Conquista, estado da Bahia, totalizando 19 palestras, 33 comunicações orais e 47 pôsteres. A terceira edição, realizada na Universidade Federal do Espírito Santo (UFES), Campus São Mateus, entre os dias 31 de outubro e 2 de novembro de 2016, contou com 179 inscrições entre pesquisadores e estudantes de pós-graduação. Estiveram presentes ao evento 126 participantes de diversos estados brasileiros, totalizando 93 trabalhos submetidos, sendo aprovadas 74 comunicações orais e três mesas temáticas.

Pode-se notar um aumento considerável do número de trabalhos que pesquisam a HEM e, desta forma, o círculo esotérico vem se fortalecendo com a criação de novos grupos de pesquisa e eventos na área.

Apresenta-se a seguir, o epistemólogo Fleck (1896-1961) e alguns de seus conceitos, que se buscou relacionar, como circulação inter e intracoletiva, com os grupos de pesquisa de Santa Catarina que investigam a HEM.

\section{Ludwik Fleck: uma aproximação com sua epistemologia}

Ludwik Fleck nasceu na cidade de Lwöw (atualmente pertencente à Ucrânia), em 11 de julho de 1896. Fez medicina pela Universidade de Jan Kazimierz (uma das maiores universidades da Ucrânia). Durante sua carreira profissional, suas investigações seguiram a linha de estudo referente à microbiologia. Atuou como professor e diretor de centros de pesquisa e laboratórios especializados. Nos últimos anos de sua vida se mudou para Ness-Ziona e trabalhou no Instituto Israelense para Pesquisa Biológica, assumindo a direção do Departamento de Patologia Experimental. Não resistiu a um segundo infarto, falecendo no dia 05 de junho de 1961, aos 65 anos. (SCHÄFER; SCHNELLE, 2010).

Além da sua vasta produção na área da medicina, Fleck também desenvolveu alguns trabalhos na área da epistemologia. Sua primeira publicação na área da teoria das ciências remonta a uma exposição apresentada em 1926, na Sociedade dos amigos da história da medicina de Lwöw, a qual foi publicada no ano seguinte numa revista polonesa sobre história e filosofia da medicina intitulada Algumas características específicas do modo médico de pensar. O segundo artigo na área da epistemologia foi publicado em 1929, intitulado Sobre a crise da 'realidade, onde aparece pela primeira vez o conceito de EP. Fleck generaliza suas proposições para além da medicina, abordando as ciências naturais, e relaciona o objeto de conhecimento no âmbito social. Esses dois trabalhos contêm as inovações teóricas que ele desenvolveu de maneira detalhada em seu livro de 1935, Gênese e desenvolvimento de um fato cientifico ${ }^{5}$. (SCHÄFER; SCHNELLE, 2010).

Em seu livro, Fleck aborda o contex to histórico-psicocultural da reação de Wassermann, usada para o diagnóstico da sífilis. Como o processo de produção de conhecimento, a investigação científica possui aspectos relativos ao meio social. O que diferencia Fleck dos demais

\footnotetext{
${ }^{5}$ No original: Entstehung und entwicklung einer wissenschaftlichen Tatsache: einführung in die Lehre vom Denkstil und Denkkollektiv.
} 
epistemólogos é a importância que ele dá à construção histórico-social do desenvolvimento da ciência e das teorias do conhecimento. Pode-se ressaltar que há uma relação entre o EP e os conceitos relevantes de um período, considerando o contexto histórico-cultural no desenvolvimento do pensamento (DELIZOICOV et al., 2002; PFUETZENREITER, 2003). Tal compreensão se deve à concepção ontológica do sujeito do conhecimento pressuposta por Fleck, segundo a qual há uma dimensão histórico-cultural que constitui o sujeito e é um dos parâmetros da produção de conhecimento. Contudo, o autor também analisa aspectos atemporais que integram a sua teoria do conhecimento. Assim, a perspectiva lógica não deve ser descartada de suas considerações epistemológicas. São várias as passagens do seu livro onde Fleck (2010) destaca o papel das conexões, ou relações, passivas, mediatizadas pelas interações do sujeito com o objeto que deseja conhecer. Assim, por exemplo, pode-se citar:

[...] encontram-se sempre no conteúdo do conhecimento outras relações que não se explicam psicologicamente (seja no plano individual, seja no coletivo), nem historicamente. Por isso, elas passam a impressão de serem relações "reais", "objetivas" ou "efetivas". Nós as denominamos de relações passivas, em oposição àquelas outras, que denominamos ativas [...].(FLECK, 2010, p. 50).

O processo de construção do conhecimento, para Fleck, se dá pela interação entre o sujeito, o objeto e a imagem, ou seja, o EP que é compartilhado pelo CP, uma estrutura triádica entre o conhecimento e suas relações. É o resultado sócio-histórico de um coletivo e está vinculado a fatores socioculturais, empíricos e lógicos. Com o intuito de elencar os conceitos elaborados por Fleck considerados importantes para a constituição de um grupo de pesquisa, explana-se o CP, como uma comunidade de pessoas portadora de um EP, ou seja, que se encontram em reciprocidade de conhecimentos, ideias e práticas. Cada coletivo é um porta-voz de uma determinada área de pensamento, um estado do saber e da cultura no seu desenvolvimento histórico. (FLECK, 2010).

É possível considerar que o CP possui uma estrutura interna, o conhecimento não é isolado, "a palavra 'conhecer' somente ganha um significado no contexto de um coletivo de pensamento” (FLECK, 2010, p. 86), compartilhando práticas, concepções, tradições e normas. Por conseguinte, o EP condiciona e regula a produção de conhecimento dentro do coletivo. Fleck define o EP como um ponto de vista, uma percepção direcionada, que possui um sistema estrutural de resistência a tudo que o contradiz, são características e problemas comuns que interessam a um coletivo. O EP exerce uma coerção de pensamento, ou seja, considerando uma e não outra maneira de perceber e agir. No entanto, o EP está em progressiva transformação, conforme argumenta Fleck (2010, p. 95), "a engrenagem das ideias e verdades somente se conserva mediante um movimento constante e efeitos recíprocos". Papel fundamental nas transformações do EP é desempenhado por problemas que o CP tem a consciência de não poder solucionar com o que é compartilhado num determinado momento histórico. Dada essa característica desses problemas o autor os denomina de complicações (FLECK, 2010). É no enfrentamento das complicações, através de mediações possibilitadas, também, e especialmente, por circulações intercoletivas que o CP propicia as transformações do EP, uma vez que "qualquer tráfego intercoletivo de pensamento traz consigo um deslocamento ou uma alteração 
dos valores de pensamento" (FLECK, 2010, p. 161). Neste sentido, algo inédito na história do conhecimento não só emerge, como exige transformações em ideias, práticas e conhecimentos compartilhados anteriormente.

O CP, conforme concebido por Fleck (2010), é composto por círculos esotérico e exotérico: o primeiro é constituído pelos especialistas e o segundo representa os leigos formados. Pode-se considerar na área da HEM, que estamos analisando, que o círculo esotérico é formado por pesquisadores que investigam e publicam na área. Já o círculo exotérico seriam os professores e pesquisadores que se interessam pelo tema, mas que, no entanto, não se dedicam à pesquisa em HEM. "Um coletivo de pensamento consiste em muitos desses círculos que se sobrepõem, e um indivíduo pertence a vários círculos exotéricos e a poucos, eventualmente a nenhum, círculo esotérico" (FLECK, 2010, p. 157).

A dinâmica que ocorre nas relações estabelecidas entre os componentes do coletivo de pensamento acontece por meio da circulação intracoletiva e da intercoletiva. A circulação intercoletiva propicia que um indivíduo pertencente a vários círculos exotéricos venha a compartilhar de um outro determinado EP, ainda não compartilhado por ele, e cujo círculo esotérico do CP correspondente difere daqueles que deram origem aos círculos exotéricos dos quais este indivíduo já compartilha. A circulação intercoletiva contribui, assim, para a extensão deste outro EP. A circulação intracoletiva, por sua vez, é fundamental para a formação e constituição de especialistas que buscam soluções para os problemas dos quais se ocupa o círculo esotérico de um determinado CP (FLECK, 2010).

De acordo com Lorenzetti, Muenchen e Slongo (2017), o interessante ao analisar a teoria do conhecimento de Fleck, está no fato de poder ser utilizada em diversos estudos de diferentes áreas da produção do conhecimento científico, desde investigações relacionadas à medicina, como em seu livro, aos estudos voltados ao ensino. A circulação inter e intracoletiva de ideias são as principais categorias de Fleck utilizadas neste trabalho. A seguir apresentamos os grupos de pesquisa de Santa Catarina que investigam a HEM.

\section{Circulação inter e intracoletiva nos Grupos de pesquisa}

Segundo Pinto (2014), a partir da década de 1960, no Brasil, o campo da História da Educação busca novos objetos, problemas e abordagens de pesquisa, por exemplo, a possibilidade de desenvolver pesquisas em educação matemática. A seguir, o autor destaca alguns fatos que contribuíram para o surgimento da área da Educação Matemática no Brasil:

[...] Movimento Escolanovista desencadeado a partir da década de 1920, o Movimento da Matemática Moderna dos anos 60, a realização de Congressos Brasileiros de Ensino de Matemática nas décadas de 50 e 60 [...] O início da década de 1970 aos primeiros anos de 1980 marca o período do nascimento da Educação Matemática, enquanto campo, não só do ensino, mas também, da pesquisa. (PINTO, 2014, p. 20).

No campo da História da educação a criação de associações científicas e de grupos de pesquisas ocorreu na década de 1980. A constituição de fóruns de discussão, nos anos de 1990, contribuiu para a circulação e divulgação da produção na área, "com implicações para 
o alargamento da pesquisa e também de estudos, no âmbito da história cultural, de temáticas como saberes, disciplinas escolares, objetos que também tem atraído pesquisadores para a história da educação matemática" (PINTO, 2014, p. 22-23).

Destaca-se, portanto, a possibilidade da ocorrência de circulação intercoletiva ocorrida entre, pelo menos, o Movimento Escolanovista e grupos de educadores no enfrentamento de problemas na implementação de um ensino da denominada Matemática Moderna (MM), que poderíamos associar a uma complicação nas práticas envolvidas, historicamente, no ensino escolar de matemática, dentre outras disciplinas. Estas práticas, hegemônicas, principalmente antes da MM, estavam em sintonia com a concepção de Educação Tradicional. O ideário desta concepção, contrariamente ao da Escola Nova, não contemplava a necessidade de uma participação ativa do aluno, implicando em uma concepção de sujeito - tanto aluno como professor - bem como de abordagens pedagógicas, distintas das que caracterizam a Educação Tradicional (LIBÂNEO, 1994).

Por sua vez, pode-se destacar o I ENAPHEM como um dos elementos que contribuiu para a circulação intracoletiva de ideias, conhecimentos e práticas que vinham sendo incrementadas na educação matemática como, por exemplo, os oriundos de problemas enfrentados pelo Movimento da Matemática Moderna, apresentados e discutidos nesse encontro, na medida em que "caracteriza-se como evento fundador que pela primeira vez, reúne a comunidade de pesquisadores de diferentes regiões do país para debater e trocar experiências investigativas de uma temática exclusiva, a história da educação matemática” (PINTO, 2014, p. 15).

[...] ao buscarmos compreender a importância da participação dos inúmeros grupos de pesquisa que no Brasil têm contribuído para a escrita da história da educação matemática e que a partir de diferentes afiliações teóricas e para além da disputa de status acadêmico, deram visibilidade à pluralidade historiográfica do evento, a uma produção de histórias singulares. Grupos que nos últimos anos têm avançado na consecução de projetos coletivos, alargado o diálogo no espaço acadêmico brasileiro e caminhado em busca de interlocuções internacionais, na tentativa de compreender representações que marcaram normativas e práticas e deram sentido a trajetórias docentes e a práticas escolares em diferentes momentos históricos. (PINTO, 2014, p. 16-17, grifo nosso).

Além de congressos, a formação em grupos de pesquisa e em cursos de pós-graduação são elementos fundamentais que propiciam, sobretudo, a circulação intracoletiva, para a extensão de novos EPs que surgem com as complicações (FLECK, 2010). A objetivação e a materialidade da produção de conhecimento nessas instâncias decorre de publicações registradas em teses, dissertações, livros, artigos em periódicos e congressos. Essas publicações possibilitam circulações intracoletiva e intercoletiva, uma vez que disseminam EPs passíveis de balizarem pesquisadores pertencentes ao círculo esotérico de determinado EP e, também, possibilitam consultas dos pesquisadores pertencentes aos círculos exotéricos de outros EPs, mas que mantêm algum nível de interlocução com as publicações.

Neste sentido, um levantamento prévio foi realizado no Diretório dos Grupos de Pesquisa no Brasil, vinculado ao Conselho Nacional de Desenvolvimento Científico e Tecnológico $(\mathrm{CNPq})$, o qual possui informações sobre os grupos de pesquisa em atividade 
no país. Primeiramente, foi feita uma busca pelo termo "história da educação matemática" aplicado aos campos: nome do grupo, linha de pesquisa ou palavra-chave da linha de pesquisa. Obtiveram-se 36 resultados de Grupos de pesquisa que investigam a HEM, vinculados a diversas instituições de ensino superior ${ }^{6}$.

Para delimitar a pesquisa foram analisados os Grupos de pesquisa de Santa Catarina, em específico: o Grupo Ensino de Ciências Naturais e Matemática da Fundação Universidade Regional de Blumenau (FURB); Grupo de Estudos e Pesquisa em Educação Matemática (GEPEM) do Instituto Federal de Santa Catarina (IFSC); Grupo de Estudos Contemporâneos e Educação Matemática (GECEM) da Universidade Federal de Santa Catarina (UFSC); e o Grupo de Pesquisa de História da Educação Matemática - Santa Catarina (GHEMAT-SC) da UFSC. Neste artigo, abordaram-se as interações, através de distintos modos, entre os pesquisadores nos grupos de Santa Catarina que possuem como linha de pesquisa a HEM.

Como mencionado, os dados foram coletados do Diretório dos Grupos de Pesquisa no Brasil do $\mathrm{CNPq}$, que permite o acesso do registro da produção científica e tecnológica de cada pesquisador cadastrado na Plataforma Lattes. A partir dessa base de dados foi criada uma rede social no software Gephi (Figura 1). Gephi é um software de código aberto para gráficos e análise de rede. Ele usa um mecanismo de renderização 3D para exibir grandes redes em tempo real e acelerar a exploração. Uma arquitetura flexível e multitarefa traz novas possibilidades para trabalhar com conjuntos de dados complexos e produzir resultados visuais valiosos. O Gephi foi usado com sucesso para estudos de caso de rede de internet e semântica (BASTIAN; HEYMANN; JACOMY, 2009).

$\mathrm{Na}$ Figura 1, os nós (as circunferências) representam os pesquisadores e estudantes que compõem cada Grupo de pesquisa de Santa Catarina que possui como linha de pesquisa a HEM. As conexões, ou seja, as arestas neste caso representam a ligação dos membros do grupo com os líderes e com as colaborações em publicações. Os maiores nós representam os atores mais influentes, neste caso, ponderou-se pelo maior número de publicações entre artigos, livros, capítulos de livros, trabalhos completos, resumos expandidos, resumos, apresentação de trabalhos e outras publicações. A ponderação foi realizada se considerando peso dois para artigos, livros e capítulos de livros publicados e peso um, para as demais categorias. As arestas representam o peso da conexão, arestas com espessura maior representam conexões mais frequentes. As redes são representações gráficas de uma determinada população, neste caso, dos Grupos de pesquisa de Santa Catarina, com seus respectivos membros que investigam a HEM. A representação dessa rede foi possível, até o momento, considerando aspectos historiográficos e sociológicos. Contudo, o levantamento de teses, dissertações e demais publicações originárias dessas redes ainda necessita ser complementado. Uma análise dessa produção possibilitará identificar semelhanças e diferenças existentes entre os vários elementos das redes de modo que se possa caracterizar os EPs e respectivos CPs que as constituem, a exemplo de Slongo e Delizoicov (2010) que analisando teses e dissertações caracterizaram EPs originários da pesquisa em Ensino de Biologia, de Lorenzetti (2008), que de modo semelhante caracterizou EPs encontrados na pesquisa em Educação Ambiental e Da Ros (2000), que ao analisar teses e dissertações em programas de pós-graduação em Saúde Pública pode caracterizar os EPs que constituem essa área.

\footnotetext{
${ }^{6}$ Disponível em: http://dgp.cnpq.br/dgp/faces/consulta/consulta_parametrizada.jsf. Acesso em: 10 jan. 2018.
} 
Hoffmann, Y. T.; Costa, D. A.; Nakamura, L. R.; Delizoicov Neto, D.

Figura 1 - Rede dos Grupos de pesquisa de Santa Catarina que investigam a HEM

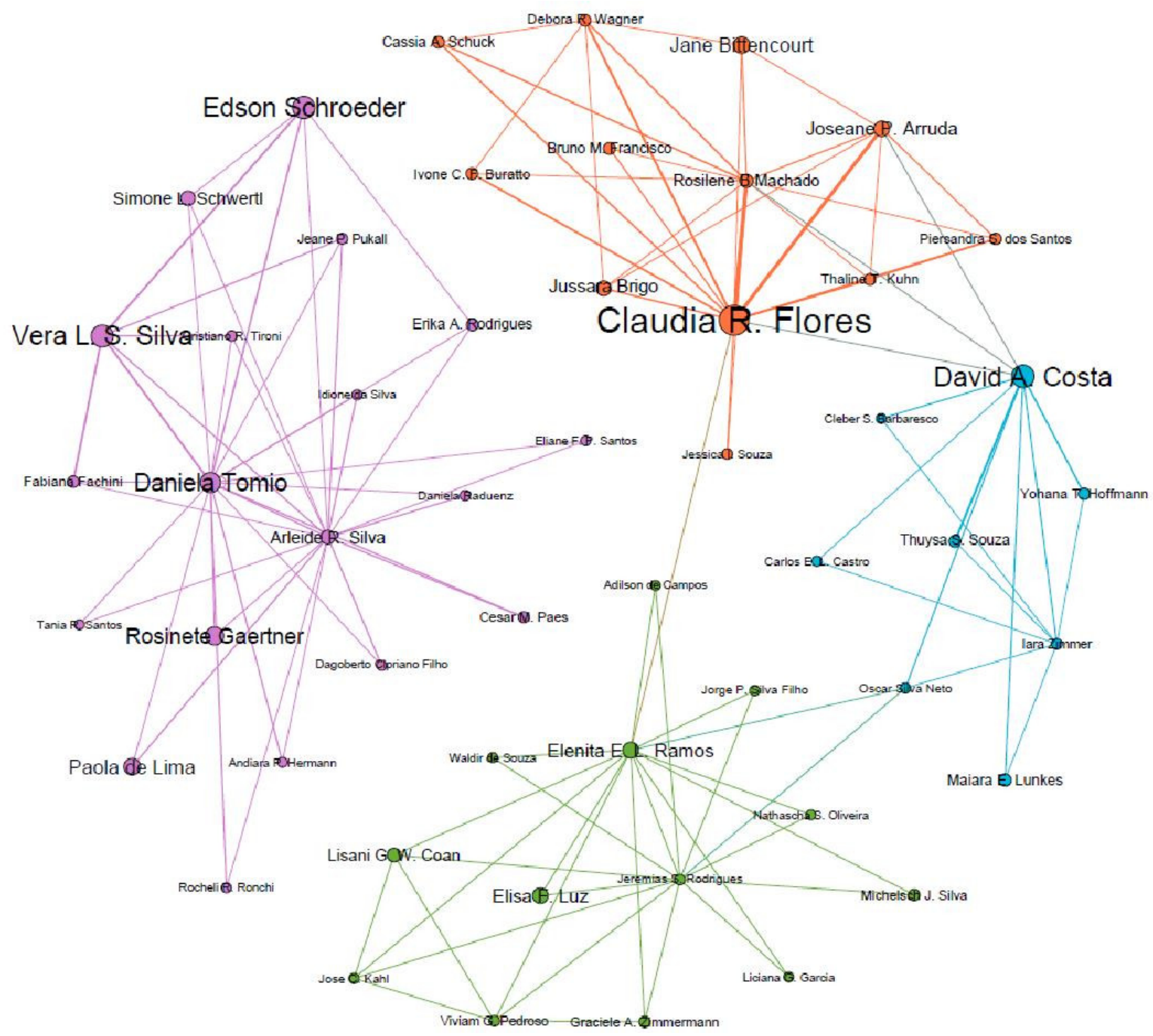

Fonte: elaborado pelos autores.

Em lilás, na figura 1, está representado o Grupo de pesquisa Ensino de Ciências Naturais e Matemática da FURB, fundado em 2010, tendo como líderes as professoras Dra. Arleide Rosa da Silva e Dra. Daniela Tomio. Possui parceria com a Fundação Educacional Barriga Verde (FEBAVE) de Santa Catarina. O grupo desenvolve pesquisas relacionadas à aprendizagem significativa, o desenvolvimento das capacidades de representação, comunicação e contextualização sociocultural, com a utilização das tecnologias da informação no processo de construção do conhecimento científico, cuja área de estudo compreende a Educação, com a linha de pesquisa em Didática das Ciências e da Matemática. 
O grupo é composto por sete pesquisadores e 12 estudantes de diferentes áreas do conhecimento, por exemplo, as grandes áreas são Ciências humanas, Ciências Exatas e da Terra, e Ciências Biológicas, no qual compreende as áreas da Educação, Psicologia, Matemática, Biologia, e Morfologia, sendo as subáreas: Educação científica e tecnológica, Educação matemática, Ensino-aprendizagem, Ensino de ciências, Formação docente, Formação de Professores, Metodologia de ensino, e, Prática docente em contextos de educação formal e não formal.

Os pesquisadores das áreas do conhecimento em Educação e Matemática, que correspondem às subáreas Ensino-aprendizagem; Formação de Professores e Educação Matemática, são os que mais possuem publicação, ou seja, representam os maiores nós dentro do grupo de pesquisa. Em relação aos demais Grupos de pesquisa de Santa Catarina que investigam a HEM, pode-se observar que o grupo Ensino de Ciências Naturais e Matemática não possui interações com os pesquisadores dos demais grupos, seja em relações de coautoria em publicações de artigos, livros e ou capítulos de livros, orientações, entre outros distintos modos. Contudo, o grupo pode estabelecer conexões com outros grupos de pesquisa, com outros círculos esotéricos dentro da diversidade das áreas de atuação dos seus membros, tanto ao nível regional, estadual, federal ou internacional que não abarcam esse levantamento.

A cor verde, na figura 1, representa o Grupo de pesquisa GEPEM do IFSC, fundado no ano de 2011, cujos líderes são os professores Dra. Elenita Eliete de Lima Ramos e Ms. Jeremias Stein Rodriguês, sendo predominante a área de Matemática. O grupo é composto por 14 pesquisadores distribuídos em diferentes linhas de pesquisa, com maior concentração na linha Educação Matemática Crítica (11). Em seguida, Ensino e Aprendizagem de Matemática (6), Registros de Representação Semiótica (4), Educação Matemática de Jovens e Adultos (2), Modelagem Matemática (2), Educação Profissional e Tecnológica (1), Formação de Professores (1), História da Educação Matemática (1), e, Matemática em contextos técnicos e tecnológicos (1).

Segundo Fleck (2010), mesmo havendo uma coerção do EP dentro do CP, os indivíduos se posicionam de maneira específica na circulação intracoletiva, pois os mesmos compartilham conhecimentos, ideias e práticas a serviço de um pensamento transpessoal, criando uma dependência entre os indivíduos. Neste grupo de pesquisa, GEPEM, os pesquisadores das áreas do conhecimento em Educação, representando as subáreas Ensino-aprendizagem, Educação a distância, Educação Matemática de Jovens e Adultos, Formação de Professores de Matemática, e, Educação Matemática, são os que mais possuem publicação, corroborando com a concentração de pesquisadores nas linhas de pesquisa em Educação Matemática Crítica e Ensino e Aprendizagem de Matemática.

Como pode ser visto na Figura 1, o GEPEM possui interações entre os pesquisadores dos Grupos de pesquisa GECEM (em cor alaranjado) e GHEMAT-SC (em cor azul) da UFSC. O elo de conexão entre o GEPEM e o GECEM é a professora Dra. Elenita Eliete de Lima Ramos que foi orientanda da professora Dra. Cláudia Regina Flores no âmbito do doutorado, além da coautoria em publicações de periódicos e eventos, principalmente na área do conhecimento em Educação Matemática de Jovens e Adultos. Segundo Löwy (1994), a socialização, neste caso dos pesquisadores dos grupos de pesquisa, em um dado EP, pensando nas investigações na HEM, pode contribuir para uma estabilidade dos grupos e dos EPs. Em relação à conexão com o GHEMAT-SC, é a participação do professor Ms. Oscar Silva Neto tanto no GEPEM, quanto no GHEMAT-SC. 
O Grupo de pesquisa GECEM da UFSC, fundado no ano de 2009, possui como líderes as professoras Dra. Cláudia Regina Flores e Dra. Rosilene Beatriz Machado. A problemática de pesquisa do grupo é direcionada aos processos de ensino e aprendizagem da produção de conhecimentos matemáticos, vinculado-se ao campo da história e da cultura. O grupo é constituído por oito pesquisadores e cinco estudantes que abarcam diferentes linhas de pesquisa: Práticas matemáticas e infância; Práticas matemáticas e narrativas sobre ensinar e aprender; Práticas socioculturais, história e matemática; e, Práticas visuais e educação matemática.

As áreas de conhecimento dos pesquisadores do Grupo são Educação e Matemática, abrangendo as subáreas Arte e Educação Matemática; Educação Matemática; EnsinoAprendizagem; Formação de Professores; Fundamentos da Educação; História, Cultura e Educação Matemática; História da Educação Matemática; História da Matemática; e, Métodos e Técnicas de Ensino.

O pesquisador com maior número de publicações no GECEM, que se destaca pelas interações, através de distintos modos, entre os pesquisadores na produção do conhecimento científico é a líder professora Dra. Cláudia Regina Flores, atuando nas áreas da Arte e Educação Matemática, História da Educação Matemática; História da Matemática, Métodos e Técnicas de Ensino; Ensino-Aprendizagem, e, Formação de Professores. As interações com o grupo GEPEM já foi anteriormente mencionada, no entanto a conexão com o GHEMATSC, ocorre pela participação do professor Dr. David Antonio da Costa no GECEM, além da coautoria em publicações de periódicos, livros e eventos, assim como em participações de bancas de defesas.

O Grupo de pesquisa GHEMAT-SC da UFSC formou-se no ano de 2017 e os líderes são os professores Dr. David Antonio da Costa e Dra. Iara Zimmer, sendo que o grupo interage diretamente com o GHEMAT Brasil no desenvolvimento de projetos coletivos. De acordo com Löwy (1994), o EP possui uma unidade tanto conceitual, quanto prática de um conhecimento específico, e possui práticas, métodos e ferramentas próprios para resolver e lidar com um problema científico.

A linha de pesquisa do GHEMAT-SC é a História da educação matemática. O grupo é constituído por três pesquisadores e cinco estudantes, tendo como objetivo investigar e produzir na área da HEM com a perspectiva da compreensão histórica do ensino e aprendizagem da matemática, da trajetória de constituição da matemática escolar e da formação de professores que ensinam matemática. O grupo também é o responsável pela Base de dados digitalizada contendo fontes de pesquisa utilizadas por todos os membros do GHEMAT Brasil, Repositório de Conteúdo Digital (RCD), em específico a comunidade História da educação matemática (L'bistoire de l'éducation mathématique). Este ambiente virtual, o RCD, está hospedado nos servidores da Universidade Federal de Santa Catarina (UFSC).

Os membros do grupo são da área da Educação, com as subáreas em Educação Matemática e História da Educação Matemática. O pesquisador com maior número de publicações no GHEMAT-SC, que se destaca pelas interações com os demais pesquisadores na produção do conhecimento científico, é o líder professor Dr. David Antonio da Costa, atuando nessas mesmas áreas do conhecimento.

Os Grupos de pesquisa de Santa Catarina que investigam a HEM se constituem como $\mathrm{CPs}$, "o coletivo de pensamento consiste em indivíduos diferentes, tendo também sua forma psíquica particular e regras particulares de comportamento” (FLECK, 2010, p. 87), no qual 
seus membros contribuem para a circulação de diversos EPs, pois são pesquisadores/estudantes que possuem formação e áreas de atuação diferentes.

A análise do movimento da produção em história da educação matemática, em termos de estreitamento do diálogo inter grupos de pesquisa, tem na realização do I ENAPHEM um fato emblemático. A proposta do Encontro demonstrou claramente a disposição dos diferentes grupos de pesquisa abrirem-se para o diálogo e promoverem trocas importantes relativamente a temas fundamentais para historiografia, em termos do "método histórico". O programa do Encontro, elaborado coletivamente, com a participação de quase trinta pesquisadores, representantes de vertentes as mais diversas da produção em história da educação matemática, tratou das diferentes bases teórico-metodológicas da pesquisa, do trato com as fontes e sua diversidade, de temáticas que envolveram histórias locais da educação matemática, história comparativa, de disciplinas escolares e acadêmicas, dentre outros assuntos na exposição e debate de uma gama enorme de problemas de pesquisa (VALENTE, 2014, p. 108).

Os integrantes do CP concordam e se adaptam à teoria e metodologia do coletivo. Essa análise dos Grupos de pesquisa de Santa Catarina que investigam a HEM buscou observar como se dão as interações, através de distintos modos, entre os pesquisadores. Ao todo foram coletadas informações de 52 pesquisadores e estudantes para criar a rede social. Optouse como ponderação, nesta análise, as áreas de conhecimento dos grupos de pesquisa, e dos membros com maiores publicações.

[...] diante da necessidade de garantir a especificidade, a originalidade do tratamento dos temas de pesquisa e as referências próprias do novo campo em constituição, é necessário restringir o diálogo com áreas em relação às quais se deseja manter autonomia, ou mesmo uma autonomia relativa. Trata-se da construção de objetos de pesquisa próprios do novo campo (VALENTE, 2014, p. 105).

Os grupos de pesquisa Ensino de Ciências Naturais e Matemática, o GEPEM e GECEM dialogam com as pesquisas que são desenvolvidas no campo da HEM, porém o grupo de pesquisa que mais realiza trabalhos e possui sua característica principal, como linha de pesquisa, é o GHEMAT-SC. Isso se dá pelos elementos constitutivos do campo de pesquisa, o qual possui aproximações dialógicas com os campos da História da educação, História da matemática, Educação matemática, entre outros.

\section{Algumas considerações}

Buscou-se neste trabalho relacionar os conceitos de Ludwik Fleck (1896-1961), como por exemplo, coletivos e estilos de pensamento, círculos exotéricos e esotéricos, e circulação 
inter e intracoletiva, utilizando como locus a área da História da educação matemática (HEM). O campo da HEM nos últimos anos vem ganhando novos adeptos. Podem-se considerar suas primeiras pesquisas a partir da interlocução de pesquisadores matemáticos interessados pela história da educação e da educação matemática, o que possivelmente implicaria na instauração de um ou mais EPs, o que pode ser caracterizado com a análise epistemológica da produção que está sendo disseminada, por exemplo, através de publicações em HEM originárias de várias fontes.

Como mencionado, foi realizado um levantamento no Diretório dos Grupos de Pesquisa no Brasil do $\mathrm{CNPq}$, que permite o acesso do registro da produção científica e tecnológica de cada pesquisador cadastrado na Plataforma Lattes. Foram selecionados os Grupos de pesquisa de Santa Catarina que investigam a HEM, em particular o grupo Ensino de Ciências Naturais e Matemática da Fundação Universidade Regional de Blumenau (FURB); Grupo de Estudos e Pesquisa em Educação Matemática (GEPEM IFSC) do Instituto Federal de Santa Catarina (IFSC); Grupo de Estudos Contemporâneos e Educação Matemática (GECEM); e, o Grupo de Pesquisa de História da Educação Matemática - Santa Catarina (GHEMAT-SC) da Universidade Federal de Santa Catarina (UFSC).

A partir dos dados coletados se criou uma rede social dos pesquisadores e estudantes dos grupos, pelo software Gephi, como ilustra a Figura 1. A visualização da rede possibilitou verificar as interações entre os membros de cada grupo, através das relações de coautoria em publicações de artigos, livros e ou capítulos de livros, orientações, entre outros distintos modos. Comprovou-se que os grupos possuem em comum a linha de pesquisa em História da educação matemática. No entanto, um levantamento de teses, dissertações e demais publicações necessita ser acrescentado para identificar e caracterizar os diferentes EPs e respectivos CPs.

Contudo cabe ressaltar outro aspecto em relação à rede social dos Grupos de pesquisa, que está exposta à temporalidade e ao caráter transitório dos grupos, ou seja, às entradas e saídas dos membros quando estudantes em nível de graduação, mestrado e doutorado. Há uma constante transformação da rede, que influencia na circulação intra e intercoletiva de ideias, práticas e conhecimentos compartilhados.

\section{Agradecimentos}

Os participantes deste estudo agradecem a contribuição e apoio à Coordenação de Aperfeiçoamento de Pessoal de Nível Superior e à Fundação de Amparo à Pesquisa e Inovação do Estado de Santa Catarina.

\section{Referências}

BASTIAN, M.; HEYMANN, S.; JACOMY, M. Gephi, an open source graph visualization and manipulation software. [S.1.]: Association for the Advancement of Artificial Intelligence, 2009. Disponível em: https://gephi.org/publications/gephi-bastian-feb09.pdf. Acesso em: 21 out. 2019. 
BRITO, A. J.; MIORIM, M. A. A institucionalização da história da educação matemática. In: GARNICA, A. V. M. (org.). Pesquisa em história da educação matemática no Brasil. São Paulo: Livraria da Física, 2016. v. 1, p. 67-92.

CONDÉ, M. L. L. Prefácio à edição brasileira: um livro e seus prefácios: de pé de página a novo clássico. In: FLECK, L. Gênese e desenvolvimento de um fato científico:

introdução à doutrina do estilo de pensamento e do coletivo de pensamento. Belo Horizonte, Fabrefactum Editora, 2010. p. xii-xvi.

DA ROS, M. A. Estilos de pensamento em saúde pública: um estudo da produção da FSP-USP e ENSP-FIOCRUZ, entre 1948 e 1994, a partir da epistemologia de Ludwik Fleck. 2000. 207 f. Tese (Doutorado em Ensino de Ciências) - Centro de Ciências da Educação, Universidade Federal de Santa Catarina, Florianópolis, 2000. Disponível em: http://www. bu.ufsc.br/teses/PEED0230-T.pdf. Acesso em: 21 out. 2019.

DELIZOICOV, D.; CASTILHO, N.; CUTOLO, L. R. A.; DA ROS, M. A.; LIMA, A. Sociogênese do conhecimento e pesquisa em ensino: contribuições a partir do referencial fleckiano. Caderno Brasileiro de Ensino de Física, Florianópolis, v. 19, n. esp., p. 52-69, jan. 2002.

FLECK, L. Gênese e desenvolvimento de um fato científico: introdução à doutrina do estilo de pensamento e do coletivo de pensamento. Belo Horizonte, Fabrefactum, 2010.

LIBÂNEO, J. C. Didática. São Paulo: Cortez, 1994.

LORENZETTTI, L. Estilos de pensamento em educação ambiental : uma análise a partir das dissertações e teses. 2008. 406 f. Tese (Doutorado em Educação Científica e Tecnológica) - Universidade Federal de Santa Catarina, Florianópolis, 2008. Disponível em: http:/ /www. tede.ufsc.br/teses/PECT0078-T.pdf. Acesso em: 21 out. 2019.

LORENZETTTI, L.; MUENCHEN, C.; SLONGO, I. I. P. A epistemologia de Fleck como referência para a pesquisa em educação em ciências no Brasil. In: ENCONTRO NACIONAL DE PESQUISA EM EDUCAÇÃO EM CIÊNCIAS., 11, 2017, Florianópolis. Anais [...]. Florianópolis: Abrapec, 2017. p. 1-9.

LÖWY, I. Fleck e a historiografia recente da pesquisa biomédica. In: PORTOCARRERO, V. (org.). Filosofia, história e sociologia das ciências I: abordagens contemporâneas. Rio de Janeiro: Editora FIOCRUZ, 1994. p. 233-249.

MARTINS, M. A. M. Estudo da evolução do ensino secundário no Brasil e no estado do Paraná com ênfase na disciplina de matemática. 1984. Dissertação (Mestrado em Educação) - Universidade Federal do Paraná, Curitiba, 1984. Disponível em: http://hdl. handle.net/1884/37365. Acesso em: 21 out. 2019.

MIORIM, M. A. O ensino de matemática: evolução e modernização. 1995. 218 f. Tese (Doutorado em Educação) - Faculdade de Educação, Universidade Estadual de Campinas, 1995. Disponível em: http://bdtd.ibict.br/vufind/Record/ CAMP_6506916d27641abb5ab21a75542a2c46. Acesso em: 21 out. 2019. 
PFUETZENREITER, M. R. Epistemologia de Ludwik Fleck como referencial para a pesquisa nas ciências aplicadas. Episteme, Porto Alegre, v. 16, p. 111-135, 2003.

PINTO, N. B. A educação matemática brasileira e a realização do primeiro encontro nacional de pesquisas em história da educação matemática: da importância do I ENAPHEM. In: VALENTE, W. R. (org.). História da educação matemática no Brasil: problemáticas de pesquisa, fontes, referências teórico-metodológicas e histórias elaboradas. São Paulo: Livraria da Física, 2014. 13-29.

SCHÄFER, L.; SCHNELLE, T. Introdução: fundamentação da perspectiva sociológica de Ludwik Fleck na teoria da ciência. In: FLECK, L. Gênese e desenvolvimento de um fato científico: introdução à doutrina do estilo de pensamento e do coletivo de pensamento. Belo Horizonte: Fabrefactum, 2010. p. 1-36.

SLONGO, I. I. P; DELIZOICOV, D. Teses e dissertações em ensino de biologia: uma análise histórico epistemológica. Investigações em Ensino de Ciências, Porto Alegre, v. 15, n. 2, p. 275-296, 2010.

VALENTE, W. R. Diálogos pertinentes da história da educação matemática: os diálogos trans, inter e intra da história da educação matemática. In: VALENTE, W. R. (org.). História da educação matemática no Brasil. São Paulo: Livraria da Física, 2014. v. 1, p. 97-116.

VALENTE, W. R. A internacionalização da pesquisa em história da educação matemática: movimentos de criação de um novo campo disciplinar. Cadernos de História da Educação, Uberlândia, v. 16, n. 3, p. 610-618, 2017. DOI: https://doi.org/10.14393/ che-v16n3-2017-3. 